\title{
Antinomien des (neuen) Urbanismus. Henri Lefebvre, die HafenCity Hamburg und die Produktion des posturbanen Raumes: eine Forschungsskizze
}

\author{
Thomas Dörfler
}

Eingegangen: 27. Mai 2010 / Angenommen: 10. März 2011 / Online publiziert: 5. April 2011

(C) Die Autor(en) 2011. Dieser Artikel ist auf Springerlink.com mit Open Access verfügbar.

\begin{abstract}
Zusammenfassung Der Artikel untersucht die Paradoxien der Stadtplanung mittels Lefebvres Raumkonzeption. Er zeigt, wie eines der prestigeträchtigsten „Waterfront“-Projekte der aktuellen Stadtplanung in Europa mit der Komplexität der Urbanität kämpft. Letztere wird dabei als spontane, nichtinstrumentelle Erfahrung urbaner Räumlichkeit verstanden, die auf Lefebvres espace vécu verweist, und die innerhalb der Rationalitäten moderner Planung kaum zu erreichen ist. Um dies zu zeigen, untersucht der Artikel empirische Daten, Bildrepräsentationen und Teile des Masterplans, um die Idee zu entwickeln, dass geplante Urbanität eine Antinomie im Žižekschen Sinne darstellt: Sie verunmöglicht Urbanität. Einige Einsichten in dieses Problem werden vorgestellt, das als ernsthafte Restriktion der - wenngleich reflektierten und gut informierten - urbanen Entwicklungsprojekte gelten kann. Indem diese Antinomien reflektiert werden, soll weiteres kritisches Denken zur Verbesserung urbaner Projekte beitragen.
\end{abstract}

Schlagwörter Stadtsoziologie · Gentrification · Lefebvre · Produktion des Raumes $\cdot$ Stadtkultur

\section{The Antinomies of (New) Urbanism. Henri Lefebvre, HafenCity Hamburg and the Production of Posturban Space. An Outline}

\begin{abstract}
The article examines some paradoxes of urban planning by referring to Lefebvre's concept of space. It shows how one of the most prestigious projects of wa-
\end{abstract}

Dr. T. Dörfler $(\square)$

Institut für Soziologie, Georg-August-Universität Göttingen,

Platz der Göttinger Sieben 3, 37073 Göttingen, Deutschland

E-Mail: thomas.doerfler@sowi.uni-goettingen.de terfront development and new urban planning in Europe struggles with the complexity of urbanity. The latter is taken as a spontaneous, non-instrumental experience of urban spaces which is strongly related to Lefebvre's espace vécu and is hardly to establish within the rationales of modern planning. To show this, the article examines empirical data, pictoral representations and pieces of the masterplan to develop the idea that planned urbanity is an antinomy in the Žižekian sense: it makes urbanity impossible. Some insights are given into this problem which is regarded as a severe restriction of - albeit reflexive and well informedurban redevelopment projects. In getting aware of these antinomies, further critical thinking on enhancing urban performances is hopefully fostered.

Keywords Urban Sociology · Gentrification - Lefebvre · Production of Space $\cdot$ Urban Culture
„Der Form des neuen Produktionsmittels, die im Anfang noch von der des alten beherrscht wird (Marx), entsprechen im Kollektivbewußtsein Bilder, in denen das Neue sich mit dem Alten durchdringt. Diese Bilder sind Wunschbilder und in ihnen sucht sich das Kollektiv die Unfertigkeit des gesellschaftlichen Produkts sowie die Mängel der gesellschaftlichen Produktionsordnung sowohl aufzuheben wie zu verklären. Daneben tritt in diesen Wunschbildern das nachdrückliche Streben hervor, sich gegen das Veraltete - das heißt aber: gegen das Jüngstvergangene - abzusetzen. Diese Tendenzen weisen die Bildphantasie, die von dem Neuen ihren Anstoß erhielt, an das Urvergangene zurück. In dem Traum, in dem jeder Epoche die ihr folgende in Bildern vor Augen tritt, erscheint die letztere vermählt mit Elementen der Urgeschichte, das heißt einer klassenlosen Gesellschaft."

Walter Benjamin, „Paris, die Hauptstadt des XIX. Jahrhunderts“ (1991 [1935]: 46 f.) 


\section{Einleitung}

Das wieder erwachte Interesse an der Stadt als Lebensort und Gestaltungsraum scheint in den Wissenschaften angekommen zu sein: Kaum eine themenrelevante Publikation verzichtet auf den Hinweis, dass es eine „Renaissance“ des Städtischen gebe, die den wichtigsten regionalen Siedlungstrend der letzten Jahrzehnte - die Suburbanisierung zumindest in Teilen rückgängig machen würde. Dergleichen gilt für die Medien und die sogenannte freie Wirtschaft, die in vielen aktuellen Projekten aggressiv mit „neuen“ städtischen Images wirbt, so etwa, um nur einige der auffallendsten Projekte hierzulande zu nennen, bei den Initiativen „Gateway Gardens Frankfurt“" , der „MediaSpree Berlin“2

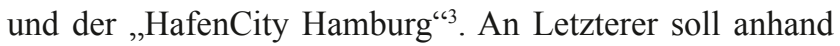
empirischen Materials die Antinomie aufgezeigt werden, einen eigentlich unplanbaren städtischen Gesamtzusammenhang (neue lebendige Stadtteile) rational planen zu wollen, was mit Bezug auf Henri Lefebvre als das Gegenteil von Urbanität aufzufassen ist.

In diesem zukunftsversprechenden Szenario sind Bürgermeister und Immobilienentwickler, Anwohner wie Firmen aus kulturellen, persönlichen oder bloßen Verwertungsinteressen vereint: Bis auf Ausnahmen haben alle diese Akteure das gemeinsame Ziel, neue urbane Umwelten in innerstädtischen „Premiumlagen“ zu schaffen. Einzig die MediaSpree Berlin sticht etwas heraus, weil sich hier bereits mehrfach und einflussreich ein Bürgerprotest formiert hat. An den Ambitionen der Entscheider mit diesem Projekt dürfte dies aber wenig ändern: Neue urbane Landschaften am Wasser sind auch in Berlin en vogue und ein Lieblingsthema von Stadtplanern.

Anders als noch in den 1970er Jahren, als die Flächensanierung das verbreitetste Mittel für die großflächige Renovierung von marodem Altbaubestand war, aber auch anders als in den 1980er und 1990er Jahren, als die behutsame Stadterneuerung antrat, die sozial desaströsen Auswirkungen abzufedern, die erstere verursachte, geht es heute um einen anderen Zugang zum Städtischen: Nicht mehr die ,Wohnungsfrage' einer durch Arbeitsmigration wachsenden Gesellschaft (und damit der Städte) ist die politische Rationalität in den Kommunen, sondern die ,Überlebensfrage" einer in die Krise geratenen postindustriellen und postfordistischen Stadt. Das alte fordistische Regime hat in der Arbeitswelt ausgedient, eine Tatsache, die seit über 30 Jahren mehr oder weniger bekannt ist. Es könnte aber sein, dass ebenso der Postfordismus an seine Grenzen stößt, den Wandel hin zu den kulturellen Ökonomien und den nun populären „kreativen Städten“ erklären zu können. Als auf

\footnotetext{
${ }^{1} \mathrm{http}: / /$ www.gateway-gardens.de.

${ }^{2} \mathrm{http} / / /$ www.mediaspree.de; Website derzeit deaktiviert.

${ }^{3}$ http://www.hafencity.com.
}

deregulierte, individuelle Arbeitskraft angewiesenes Produktionsparadigma in einer wissensbasierten Ökonomie legen solche Städte besonderen Wert auf bestimmte räumliche settings, welche Urbanität und Innovation für bestimmte Milieus versprechen: jene geradezu magisch repetierten „kreativen Räume“ lisierung der Arbeit vorantreibt, kommt es anscheinend zeitgleich, aber räumlich divergent, zu Reterritorialisierungen vor allem in den Metropolen der ersten Welt (vgl. die Arbeiten von Sassen zur Global City), die gerade nicht global ubiquitär funktionieren, sondern lokal äußerst selektiv. ${ }^{5}$ In diesem Sinne ist die oft beschworene "Standortkonkurrenz" der Städte um „Kreative Köpfe“ als Indiz bestimmter Relokalisierungen zu sehen, die im postfordistischen Paradigma zunächst so nicht vorgesehen waren: produziert und abgewandert werden konnte global, weshalb es ja gerade zum downsizing von Belegschaft und Löhnen kommen konnte. Die Creative Class (Florida 2004) hat zudem ein anderes Druckmittel: ,Schafft Ihr uns nicht entsprechende Umwelten, dann kommen wir als High Potentials und Bestverdiener erst gar nicht in Eure langweilige Stadt. ${ }^{6}$ Oder, wie Florida (2004: xxviii) schreibt, kommt es sogar vor, dass Firmen den kreativen Menschen hinterherzögen. In ökonomischer Hinsicht scheint diese Krise deshalb die Krise einer kapitalistischen Urbanisierung im globalen Maßstab (Harvey 1991; Harvey 2007) zu sein, weil genau dieser globale Rahmen seit rund 20 Jahren den Städten neue Formen der governance aufzwingt. ${ }^{\text {? }}$

\section{Der symbolische Mehrwert des Urbanen}

Mit selbst gesetztem und neu in Stellung gebrachtem kulturellen Kapital soll der Marktwert der Städte als Investitions-, Wohn- und Arbeitsort unter den Bedingungen einer nunimaginierten global-urbanen Standortkonkurrenz gesteigert werden. Dies verschiebt aber die Bedingungen für die Analyse des Prozesses, wenn gebaute Umwelt und mit ihr soziale settings gewissermaßen als Produktionsfaktoren ins Spiel kommen, will man sie nicht nur als zunehmende Kapi-

\footnotetext{
${ }^{4}$ Die Literatur dazu ist - im Gegensatz zur inhaltlichen und methodologischen Klärung des Begriffs - schier unübersichtlich und die Verwendungsweisen sind äußerst heterogen. Als eine Übersichtsstudie dazu aus Sicht der Raumplanung vgl. ILS (2008).

${ }^{5} \mathrm{Vgl}$. aber ebenso für eine frühe Kritik an der ,postmodernen“ Kulturalisierungsthese und mit Hinweisen auf „materialistische“ Relokalisierungen Harvey (1989: 190 ff.), der damit ein plumpes Verständnis der Globalisierungsprozesse als einfache „Auflösung von modernen Raum- und Zeitstrukturen“" zurückwies.

${ }^{6}$ Vgl. zum „kreativen“ Zeitmanagement heutiger Beschäftigungsverhältnisse als frühe Studie dazu Hörning/Gerhard/Michailow (1990: $48 \mathrm{ff}$.).

${ }^{7}$ Nach Lefebvre (1972a: 48) wurde bereits 1970 „,das Städtische [...] zur Episteme der Zeit“.
} 
talisierung begreifen, sondern als Teil neuer Regierungsformen urbaner Gesellschaften. Wo in den 1990er Jahren (und bisweilen auch noch heute) die Perspektive vorherrschte, das ,neoliberale“ Kapital suche sich hier schlicht neue Einsatzmärkte (was es selbstverständlich auch tut), möchte ich im Folgenden zeigen, dass die neuen urbanen Inwertsetzungslogiken weder im ökonomischen Paradigma alleine aufgehen noch lediglich durch „Diskurse“ etablierte Zeichenwelten sind, sondern gewissermaßen ein Drittes: eine kapitalisierte und kapitalisierende Raumpraxis, die ebenso zeichen- wie wissens- und ökonomiebasiert vorgehen muss, um erfolgreich zu sein, denn jede Kapitalisierung benötigt einen ,symbolischen Mehrwert', ohne den diese als imagokultureller Überbau („Globalisierung“, „, freie Marktwirtschaft") nicht funktionieren würde.

„Symbolischer Mehrwert“ bedeutet nicht, dass dieser Wert nur aus Spiegelfechterei besteht, dass er ein überflüssiges Beiwerk und keine Realie sei, sondern dass seine Realisierung an symbolische Formen und deren inhärenten imaginär-utopischen Gehalt strukturell und konstitutiv geknüpft ist (Bebilderungen, Texte, Semantiken, Legitimationsideologien). ${ }^{8}$ Der ökonomische Erfolg solcher Begriffe ist mit dem Potenzial verknüpft, als Zeichen Sinnstiftungen wie die Erfüllung von Hoffnungen und Wünschen oder auch Deutungsmacht ausüben zu können. Ein solcher Mehrwert verhilft zu größerer Überzeugungskraft in den erwähnten neuen governance-Strukturen, die die realen - meist profanen - Bedingungen nicht herzustellen in der Lage wären, weswegen Dokumente, die die Gegenwart und Zukunft unserer Gesellschaften beschreiben sollen, voll von solchen ,imaginären“ Begrifflichkeiten sind („Netzwerkgesellschaft“, „Digitaler Kapitalismus“ etc.). Im Falle der Globalisierung funktioniert sie sogar als Drohkulisse, um (kommunale) Politik unter Druck zu setzen und in gewissem Sinne ökonomisch zu erpressen („Bargaining-Strategie“, vgl. Trinczek 1999: 69 f.) - ganz gleich, wie real Standortverlagerungsstrategien tatsächlich sind.

Der symbolische Mehrwert, der die kommunalen Entwicklungsszenarien dabei begleitet und in diesem Sinne utopisch überhöht, heißt heute Kreativität. Sie ist nahezu zum Allheilmittel in Politik und Planung geronnen, wenn es darum geht, für finanziell geschwächte Städte und Kommunen Zukunftsvisionen zu entwickeln. Reckwitz begreift dies als Imperativ zur Eigeninitiative, sich ,kreativ' zu vermarkten, weswegen er diesen Zug als „Selbstkulturalisierung“ des Städtischen beschreibt: „[Sie] setzt eine Selbstbeobachtung der Stadt als ein kulturelles Gebilde, das heißt als Trägerin spezifischer Symbole, Zeichen und Praktiken voraus, und zwar in Differenz zu anderen Städten. In dieser Selbstbeobachtung kann nun alles kulturell relevant werden, einschließ-

${ }^{8}$ Zum utopischen Gehalt des Marktliberalismus vgl. z. B. Žižek (2009: $56 \mathrm{ff}$.). lich bisher banal oder sogar problematisch erscheinender Phänomene oder das, was für selbstverständlich gehalten wurde: naturräumliche Gegebenheiten, Industriedenkmäler, lokale Bräuche, ehemalige Stadtbewohner durchaus zweifelhafter Berühmtheit" (Reckwitz 2009: 7 f.).

Da Städte als Standorte industrieller Produktion zumindest auf der imaginären Ebene des Stadtmarketing immer weniger eine zukunftsträchtige Form der Ökonomie darstellen, ${ }^{9}$ sind sie also angehalten, sich kulturell zu vermarkten. Damit einher geht aber eine Änderung der Regierungsform der Stadt (Reckwitz 2009: 4 und 8), also der governance des Städtischen, welche nun nicht nur ökonomisch induzierte Standortpolitik ist, sondern „Biopolitik“ (Foucault 1999: 286) um „die besten Köpfe“ bei gleichzeitiger Exklusion bzw. Produktion ${ }^{10}$ einer urban underclass ${ }^{11}$ : Aus urban governance wird so die „Gouvernementalität“ einer selektivierenden Posturbanität, wie ich im Weiteren zeigen möchte.

Diese Tendenz ist also in der wissenschaftlichen Debatte bereits bemerkt worden und firmiert derzeit prominent unter der Analyse der „Eigenlogik“ (Berking/Löw 2008; Löw 2009), der erwähnten „Selbstkulturalisierung“ (Reckwitz 2009) oder der governance des Städtischen. Ich möchte dem im Folgenden eine weitere Perspektive hinzufügen, diejenige einer neu formulierten Lefebvreschen Kritik an den Antinomien des gegenwärtigen Urbanismus in Politik und Planung: die gouvernementale, posturbane Stadt. Anhand der Untersuchung empirischen Materials zur HafenCity Hamburg soll es im Folgenden um die als Antinomie gehandelte urbane Phantasie gehen, Urbanität planen zu wollen und damit die Realisierung derselben zu verunmöglichen.

\footnotetext{
${ }^{9}$ Dies gilt ungeachtet der realiter noch vorhandenen Produktionsstätten in wichtigen Branchen. Allerdings bemerkt man auch hier vereinzelt eine „Postmodernisierung“ vor allem bei stark medien- und imageaffinen Branchen wie der Automobilindustrie, wie die Entwürfe einiger „Stararchitekten“ für ihre Büros und Fabriken nahelegen. Im Endeffekt ist aber die These vom Ende der Industriearbeit und die mit ihr einhergehenden Verschiebungen im politischen Diskurs selbst Teil jenes ,symbolischen Mehrwertes', den einige Städte erzeugen wollen, indem sie sich explizit von der Arbeitertradition verabschieden respektive sie kulturalisieren; vgl. z. B. die „Europäische Kulturhauptstadt Ruhr.2010“ oder - für einen frühen Fall - Glasgows Umschreibung der Arbeitertradition in Mitchell (2000: 3 ff.).

${ }^{10} \mathrm{Zu}$ area bans als eine solche urbane Exklusionspolitik im Sinne des Ausschlusses nun ,problematisch“ gewordener Subjekte vgl. Belina (2007: 322 f.): Bestimmte Subjekte werden dahingehend kontrolliert, ob sie in bestimmte Gebiete ,passen“. Wenn nicht, kann ihnen aufgrund ihres Aussehens, ihrer vermuteten Gefahr für die Sicherheit, ihres Alkoholisierungsgrades, ihrer räumlichen Aufenthaltspraxis etc. der Zugang zu solch einem Gebiet mittels eines area bans verwehrt werden. Dies kann paradoxerweise sogar zu Situationen führen, dass Anwohner mindestens vorübergehend nicht mehr in ihre Wohnungen können, weil sie als „gefährlich“ für die Kontrollinstanzen dieses Raumregimes gelten.

${ }^{11}$ Vgl. zur Diskussion dazu Wacquant (2008) oder Wilson (2009).
} 


\section{Auftakt}

Gesetzt also den Fall, dass wir ein weitverbreitetes (neues) Interesse an urbanen Lebenswelten annehmen können, so stellen sich folgende entscheidenden Fragen: Woher rührt dieses neuerliche Interesse und wer - im Sinne einer soziostruktuellen Analyse - hegt dieses überhaupt? Was ist Urbanität und warum sind die oben geschilderten Szenarien möglicherweise ,posturban“? Und nicht zuletzt: gibt es ebenso andere Vorstellungen von urbanem Leben, auch und gerade im Hinblick auf manchen Protest, der die Initiativen begleitet? An der Renaissance des Städtischen sind deshalb zwei Dinge interessant und lohnen einer genaueren Betrachtung:

Eine angenommene Reurbanisierung als nennenswerte Beschäftigten- und Bevölkerungszunahme in den Städten hat keine statistische Grundlage, das heißt, es gibt derzeit in keiner Stadt Europas Hinweise darauf, dass es zu einer erneuten und nennenswerten Verdichtung innerstädtischer Gebiete durch Zuzug käme (Köppen 2011: 286 f.). ${ }^{12}$ Wenn sich aber keine quantitativen Hinweise auf Reurbanisierungsprozesse finden lassen, so muss das Interesse am Urbanen zweifelsohne qualitativ begründet sein: eine neuerdings erscheinende (oder: zu Tage tretende) Wertschätzung innerstädtischer Lebensweisen in bestimmten Kreisen, ganz gleich, was die jeweilige Klientel darunter verstehen mag.

Der Artikel möchte sich von dieser Ausgangslage aus dem derzeit vielleicht prominentesten Beispiel einer Stadtumbaumaßnahme (eigentlich: Neubau) in Deutschland widmen, der HafenCity Hamburg und deren Verortung im stadtpolitischen Kontext. Es soll ersichtlich werden, warum dieses Projekt im Rahmen vielfältiger Hamburger Versuche anzusiedeln ist, die Stadt als kosmopolitane Seemetropole neu zu erfinden. Diese Strategie nenne ich ,posturban', weil die Imperative der Planung paradoxerweise auf das Gegenteil dessen hinauszulaufen drohen, was angestrebt ist. Grundlage der Analyse soll Henri Lefebvres Konzeption des Urbanen sein, um die ,Produktion“ der HafenCity als (an der Oberfläche) widerspruchinkorporierendes, (post-) urbanes Utopia zu begreifen. Der Beitrag schließt mit einem Fazit zu dem dieser Phantasmagorie inhärenten sozialen Antagonismus.

\footnotetext{
${ }^{12}$ Damit ist nicht der feststellbare Trend zum Rückzug in die Kernstädte gemeint, der sich sehr wohl in kleinem Umfang für nahezu alle großen deutschen Städte ausmachen lässt. Allein, für eine Reurbanisierung im engen Sinne des Wortes reicht dieser noch nicht aus (vgl. Mai/Schlömer 2007; Köppen 2008). Die Reurbanisierungsthese zielt damit am Problem der ,Renaissance des Städtischen` und der Posturbanität vorbei.
}

\section{Befunde}

Die Stadt Hamburg zeichnet sich dadurch aus, dass sie - wie einige andere Metropolen auch - seit Jahrzehnten städtische Entwicklungsleitlinien formuliert, die die jeweilige Senatsperiode als Rahmenpläne begleiten. Konkret wurde dies zuerst 1983 umgesetzt, als die SPD-Regierung unter dem Bürgermeister Klaus von Dohnanyi das „Unternehmen Hamburg" als Leitbild initiierte und dies - in der Diktion des jeweils geltenden politischen Jargons - bis heute gängige Praxis wurde. Von Dohnanyi erkannte bereits damals „ein immer größeres Gewicht für die Standortentscheidung - ich sage das einmal so - einer neuen Intelligenz" ${ }^{\prime 13}$ Diese Überzeugung wurde seitdem den jeweiligen politischen Zeiten angepasst und beibehalten. Hinsichtlich des postfordistischen Szenarios, wie es eingangs beschrieben wurde, positioniert sich Hamburg in der Zwischenzeit deswegen folgendermaßen: ${ }^{14}$

Rahmenbedingungen ändern sich fortlaufend. Anschaulich wird dies in der aktuellen Wirtschaftskrise: Es reicht nicht mehr aus, allein auf die traditionellen Stärken der Stadt zu setzen; denn gerade Hafen, Handel und Außenwirtschaft sind in immer stärker werdendem $\mathrm{Maß}$ globalwirtschaftlichen Zyklen und Störungen ausgesetzt. Auch deshalb ist es wichtig, dass Hamburg sich ergänzend neue Entwicklungsbereiche wie etwa die Kreativwirtschaft, den Bereich regenerativer Energien oder die Gesundheitswirtschaft noch stärker erschließen muss. ${ }^{15}$

Diese Ansprüche galten von Anbeginn an auch für das Großprojekt HafenCity, dessen Masterplan derzeit zehnjähriges Jubiläum feiert. ${ }^{16}$ Früh begriffen hier die zuständigen Personen, dass man mit ihr nicht nur Boden für den Innenstadtbereich hinzugewinnt - eine Tatsache, deren Ausmaß alleine schon spektakulär wäre, sondern vor allem die Möglichkeit bekommt, urbane Zukunftsvisionen mitten in Hamburg konkret werden zu lassen. Aus diesem Grunde sind nahezu alle Teile dieses Viertels geprägt vom symbolischen Kapital in „utopischer“ Hinsicht, wie es skizziert wurde. ${ }^{17}$

\footnotetext{
${ }^{13}$ Zitiert nach Fischer/Jörg: Exklusiv wohnen und Arbeiten auf ,m Kiez“ in: Broschüre zum Film „Empire St. Pauli“; online unter http:// www.empire-stpauli.de/file/broschuere_web.pdf (letzter Zugriff am 14.05.2010).

${ }^{14}$ Einzusehen unter http://www.hamburg.de/leitbild-leitprojekte (letzter Zugriff am 02.05.2010).

${ }^{15} \mathrm{http}: / / w w w . h a m b u r g . d e /$ leitbild (letzter Zugriff am 10.05.2010).

${ }^{16}$ HafenCity Hamburg News, Extrabeilage, März 2010.

${ }^{17}$ Nicht vergessen werde sollte, dass Bourdieu das symbolische Kapital zuerst als Weigerung der Anerkennung ,der , objektive[n] 'Wahrheit der ,ökonomischen " Praktiken" konzipierte, also als systematische Verschleierung des blanken ökonomischen Kalküls, wie es hier anklingt, wenn auch auf eine andere Wirtschaftsform bezogen (Bourdieu 1999:
} 
Der westliche Teil der HafenCity (Dalmannkai, Sandtorkai, Grasbrook) will in Namensgebung und Vermarktung ein mediterranes Lebensgefühl evozieren, das zudem an die Tradition der Entdecker (und damit deren Offenheit für Neues) in Europas Geschichte anknüpfen soll (,Marco-PoloTower" und „Magellan-Terrassen“). Das mittlere „Überseequartier“ lässt mit der „HafenCity Universität (HCU)“, dem „Science Center“, dem „Cruise Center“ und seinen Straßennamen („San Francisco-Straße“, „Shanghai-Allee“) an Internationalität und eine neue Wissenskultur denken, wie dies auch Strategie der Entwickler ist: „Als Ausdruck der Wissensgesellschaft wird die HafenCity auch Standort von Institutionen, die sich explizit mit Wissensentwicklung und

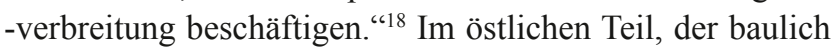
bislang kaum in Angriff genommen wurde, werden ebenso große Vorbilder bemüht, wenn etwa der „Chicago Square“ eine landmark architecture-Funktion wahrnehmen und ein „markantes Eingangstor"19 schaffen soll, das Hamburgs Partnerstadt gewidmet ist.

Was das Projekt also neben seiner beeindruckenden Größe besonders interessant macht, ist die Tatsache, dass man hier eine riesige tabula rasa vorfand und keinen Altbestand, den man erst mühsam nach Denkmalschutzrichtlinien hätte rekonstruieren oder doch zumindest hätte einbinden müssen; von Alt-Bewohnern ganz zu schweigen, deren Protest bei einer solchen Sanierung gewiss gewesen wäre. Stattdessen konnte hier komplett neu und anders entworfen werden, als das bisher in innenstädtischen Lagen möglich war. Die HafenCity könnte aus diesem Grunde gewissermaßen zur ,Stunde null' des Posturbanismus in Deutschland werden, weil sie den Initiationspunkt vermarkteter (,neuer“) Urbanität darstellt. Ebenso entscheidend ist aber, dass sich hier die derzeitigen in Deutschland populären urbanen Phantasien manifestieren: Obwohl ähnliche Bestrebungen andernorts in ähnlichem Maßstab umgesetzt werden (Bremen) und der Westhafen in Frankfurt am Main architektonisch als Vorbote der HafenCity gelten kann, so sind doch das Vorgehen und der Anspruch des Projektes faszinierend, vor allem im Hin-

215). Symbolisches Kapital ist demnach die Sublimierung profanen Verwertungsstrebens in ein Feld legitimer sozialer Anerkennung, weil das „blanke“ ökonomische Profitinteresse verpönt ist. In diesem Sinne kann hier die Brücke zu unseren „modernen“ Gesellschaften geschlagen werden: Symbolisches Kapital entsteht unter anderem dann, wenn (verpöntes) ökonomisches Profitstreben in sozial gebilligte und gratifizierte Anerkennung transformiert werden kann, etwa durch Spendengalas etc. In diesem Sinne ist die hier versuchte Transformation von ökonomisch-fiskalischen Motiven einer in die Krise geratenen fordistischen Stadt in anerkannte postfordistische (,kulturalisierte") Formen der Anerkennung symbolisches Kapital.

${ }^{18}$ Interview mit dem damaligen Vorsitzenden der Geschäftsführung Jürgen Bruns-Berentelg in „Standort“ - Zeitschrift für angewandte Geographie (2008: 40).

${ }^{19} \mathrm{http} / /$ www.hafencity.com/de/quartiere.html (letzter Zugriff am 11.05.2010) blick auf die Ambitionen in Politik und Planung, die damit einhergehen. An der HafenCity, so meine These, lassen sich bereits jetzt die - durchaus problematischen - Aspekte zukünftiger Stadtgesellschaften erkennen.

Im Weiteren ist deshalb zu zeigen, welche Aspekte urbanen Lebens in diesen Modellen repräsentiert sind (und welche nicht) und dies aus welchen Gründen. Nur dann wird verständlich, warum solche städtischen Gebiete „posturban“ genannt werden könnten. In diesem Sinne benötigt es vor allem eine adäquate Theorie, um diesen Prozess und seine Folgen richtig einschätzen zu können: Ich möchte deshalb auf die Urbanitätstheorie von Henri Lefebvre zurückgreifen, um diesen Sachverhalt zu erhellen.

\section{Was ist „Urbanität"? - eine Lefebvresche Antwort}

Einige stadtsoziologische Schriften Henri Lefebvres sollen im Folgenden Auskunft geben, um die darin entwickelte Perspektive auf Urbanität als sozioräumliches, historischmaterielles Ensemble zu verstehen.

Ausgangspunkt von Lefebvres Überlegungen ist die später so benannte „Krise der Städte“, die er bereits vor der Konjunktur dieser Metapher thematisierte. Die Arbeiten zur „Kritik des Alltagslebens“ seit den 1950er Jahren bilden eine Vorstufe zu den dann folgenden Beiträgen zum modernen (sozialen) Städtebau mit seinen neu angelegten Großwohnsiedlungen und den Folgen dieser sozialräumlichen Modernisierung für die Bewohner (Lefebvre 1974; Lefebvre 1975 [französische Originale 1958 und 1962]; Lefebvre 1972b [französisches Original 1968]; Lefebvre 1978 [französisches Original 1962]). Diese Linie gipfelt gewissermaßen in seinem bis heute einflussreichen und wiederentdeckten Werk „La révolution urbaine“, dessen Titel (inklusive des Autorennamens) bezeichnenderweise falsch ins Deutsche übertragen wurde, dadurch aber einen Einblick in die begrenzten Rezeptionsmöglichkeiten damals vorherrschender stadtplanerischer und soziologischer Zugänge zum Urbanen gibt: „Die Revolution der Städte“ hieß die deutsche Ausgabe. Hier wird bereits im Titel deutlich, wodurch sich die Lefebvresche Perspektive vom hierzulande gepflegten Blick auf das Urbane auszeichnet: Steht dort das Prozesshafte und qualitativ Verändernde im Vordergrund, ist es hierzulande die statische und raumcontainerhaft gedachte feste Form der „Stadt“ (dazu Lefebvre 1972a: 125 ff.).

Er entwickelte eine materialistische Raumtheorie, die „Raum“ nicht lediglich als Projektionsfläche bestimmter gesellschaftlicher Konflikte (beispielsweise Raumplanung vs. Ökologie) konzipiert. Vielmehr ist Raum - auf den bestimmenden Artikel wird bewusst verzichtet - bei Lefebvre ein imaginär-materialistischer Zusammenhang, der eine räumliche Praxis verdeutlichen soll, die sich nicht lediglich auf Physis bezieht (wenngleich das für ihn als materialis- 
Abb. 1 Die „Produktion des Raumes" nach Henri Lefebvre. (Quelle: Eigener Entwurf nach Schmid (2005))

\section{Die »Produktion des Raumes« nach Henri Lefebvre}

Räumliche Praxis:

Relationen, Körper, Netzwerke, Beziehungen: die „cadre de vie“ (Lebens-

Politik; aber auch im Alltag.

Zeichenvermittelt hergestellter, ,gedanklich konzipierter Raum": Karten, Pläne, Urbanitätsphantasien als Images. welt).

Urbane Wirklichkeit als Praxisform in Architektur, Städtebau, Alltagsleben.

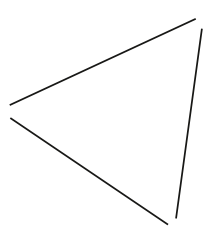

An Subjekt/Körper gebunden als notwendiges Erfahrungsmedium.
Räume der Repräsentation:

\author{
„Streben auf System nichtverbaler \\ Symbole und Zeichen hin": gesellschaft- \\ lich Imaginäres, räumliches Unbewusstes.

\begin{abstract}
Phantasmagorien, etwa in „zuhause“, „urban“.

In Poesie, Metaphern, Kämpfen um Stadtteile formulierte imaginäre Raumbezüge: „Freiraum“, „Cyberspace“ etc.
\end{abstract} \\ Die durch Zeichen mitkommunizierten
}

tischen Theoretiker unabdingbar ist), sondern auch auf die Konzeptionen und Perzeptionen von Raum als gesellschaftlicher „Umgang“ damit. Erst dieser Umgang ist praktisch und damit wirklich, weshalb es für Raumanalysen sinnvoll ist, auf diese Praktiken der Herstellung zu achten: hier werden Städte und Stadtgesellschaften ,gemacht“. Dieses Kräfteverhältnis konzipiert er zum ersten Mal synthetisiert in $L a$ production de l'espace mittels der Triade des espace perçu, des espace conçu und des espace vécu (Lefebvre 2000: 81 und 220 ff.), was sich durch „Räumliche Praxis“ (perçu), „Repräsentationen des Raumes“ (conçu) sowie „Räume der Repräsentation“ (vécu) übersetzen lässt (Schmid 2005: 210 f., 216 f. und 222 f.; vgl. Abb. 1).

Räumliche Phänomene - Lefebvre denkt hier fast ausschließlich an die Auswirkungen der „,vollständigen Urbanisierung“, wie er sie in der „Revolution der Städte“ thematisiert (Lefebvre 1972a: 7) -, die sich im Alltag ebenso wie in staatlich-institutionellen Zusammenhängen finden, lassen sich demnach innerhalb dieser drei Register analysieren. Sie bilden den „Raum der Gesellschaft“, also die aus der sozialen Praxis (Produktions- und Reproduktionsbedingungen) einer Gesellschaft hervorgehenden räumlichen Konsequenzen von Vergesellschaftung (und vor allem deren Antagonismen). Der Entwicklungsstand, also die Entwicklung der Produktivkräfte bzw. die Akkumulationsregime, haben maßgebliche Auswirkungen auf die Ausformung (sozial-)räumlicher Phänomene.

Jener „Entwicklungsstand“ und seine charakteristischen „Raumproduktionen“ (Benjamins „Passagen“ im 19. Jahrhundert (Benjamin 1991) oder „Malls“ heutzutage) sollten aber nicht lediglich als Fortsetzung von Kapitalisierung,
Neoliberalisierung o. ä. begriffen werden. ${ }^{20}$ Es gibt vielmehr auch auf der administrativen, auf der alltäglichen wie auf der kognitiven Ebene ,Entsprechungen' (Praktiken), ohne die die (kapitalistische) Vergesellschaftung und damit auch deren Verräumlichung nicht funktionieren kann. Lefebvre rückt damit in die Nähe der Regulationstheoretiker, für die vor allem das Politische ein abgeleiteter, aber notwendiger Bereich der Akkumulation ist, ohne dessen Ressourcen (Gesetze, Privilegien, Entscheidungsinstanzen) die Verwertungsinteressen nicht in dem Maße voranschreiten könnten, wie sie es tun. Die Politik ist in dieser Hinsicht ein Anhängsel der Ökonomie, ohne jedoch davon ,determiniert $^{`} \mathrm{zu}$ sein, wie eine vulgärmarxistische Lesart behaupten könnte. Lefebvre geht darüber hinaus und begreift auch konzeptionelle Repräsentationen von Raum (Architektur) oder Stadtimages (,Bilder im Kopf', Medienbilder) als solche notwendigen Supplementierungen für die Inwertsetzung von Raum.

Damit wird der Vorteil dieses Zugangs zum Räumlichen deutlich: Zum einen ist es möglich, die im Kapitalismus gewissermaßen systemhaft notwendig vonstattengehenden Stadtumbau- und Modernisierungsmaßnahmen nicht nur in ,klassischer' Weise als Ausdruck einer kapitalistischen Praxis der Verfügbarmachung von Wohn- oder Büroraum zur Wertsteigerung zu betrachten, sondern es gerät auch die administrative und kognitive (und damit gesellschaftlich beeinflussbare) Ebene des Politischen in den Blick, das diesen Prozess ideologisch und praktisch stützen muss. Dies

\footnotetext{
${ }^{20}$ Und dies nicht nur, weil der Begriff zu der Zeit, als Lefebvre dies konzipierte, noch nicht existierte.
} 
schärft das Verständnis dafür, wie etwa bestimmte gesellschaftliche Instanzen, bestimmte Diskurse in den Stadtgesellschaften oder imaginative Korrespondenzen in den Medien daran teilhaben, Raum zu produzieren, der dadurch ein ,Ausdruck' im Sinne einer Repräsentation desselben ist. Ist dieser Raum noch nicht existent (wie im Falle der HafenCity), so haben wir in den Plänen, den Legitimationsdiskursen und in den gestalterisch-praktischen Umsetzungen für bestimmte soziale Schichten (besser: Milieus) die jeweilige urbane Utopie in nuce vor uns: in den Bildern, Stadtdiskursen, Begrifflichkeiten, also in den Semantiken des Urbanen lassen sich diese aufspüren und aufzeigen, wie der folgende empirische Teil des Artikels exemplarisch zeigen will.

Urbanität im Lefebvreschen Sinne ist also produzierter städtischer Raum, der deswegen „städtisch“ ist, weil er modernistisch die gesamten Produktions- und Reproduktionsbedingungen einer Gesellschaft urbanisiert. Selbst (vormoderne) Landwirtschaft wird in dieser Hinsicht urban, da sie spätestens seit dem 19. Jahrhundert eine industrielle, auf urbaner Technik basierende und auf urbane Abnehmer abzielende kapitalintensive Tätigkeit ist, die ebenso Raum produziert - urbanen Raum in dialektischer Abhängigkeit zur Stadt. Gleiches gilt für die besprochenen Beispiele: Sie sind deswegen urban, weil sie bestehende ,traditionelle", mittlerweile anti-urbane Ensembles (alte Industrieanlagen, Stadtbrachen etc.) als städtischen Raum neu in Wert setzen - und dies in den drei genannten Registern des Raumes.

Diese aber, das ist die eigentümliche Dialektik bei Lefebvre, widersetzen sich einer rein instrumentellen Anwendung, da mindestens ein Teil der Raumproduktionen, der „gelebte" Raum espace vécu, auf latenten (unbewussten) Erfahrungen aufbaut, die das Subjekt (wir, die Stadtbewohner) für die Aufrechterhaltung unserer Subjektivität benötigen: die Vorstellung des Spontanen, Nicht-Instrumentellen, hier in räumlicher Hinsicht gedacht (sich frei in der Stadt bewegen können, z. B. im Gegensatz zum ,einengenden Dorf'). Mit anderen Worten: keine semantische respektive symbolische Raumproduktion (z. B. eine Vorstellung von „Zuhause“, „Cosmopolit“, „Heimat“, „globales Dorf“ oder eben „HafenCity“ als semantischer Konnex von maritimer Tradition und innerstädtischer Zentralitätsfunktion) operiert ohne den phantasmagorischen Anteil subjektiver (gewählter, gewünschter) Zugehörigkeit - für diejenigen, die diese Phantasien hegen (können) selbstverständlich. Alle solche Raumproduktionen begleitet deshalb die Vorstellung, dass hier ,spontan' neuer Raum erschlossen wird.

An der Antithese kann man dies besonders deutlich sehen: Wird instrumenteller Raum geschaffen, der dennoch solche Phantasmagorien eines freien Zugangs stützen muss, dann verliert er wegen der fehlenden dort zu realisierenden Spontanität seine Attraktivität (z. B. von den Kommunen offiziell zur Verfügung gestellte Graffitiwände). Als „instrumentellen Raum" möchte ich deshalb hier eine solche Raum- produktion bezeichnen, die den Anteil des espace vécu als gelebten Raum, jenseits von planerischen oder zweckmäßigen Aspekten, leugnen respektive camouflieren muss durch etwas, das diese Raumproduktion für gelebten Raum hält, ohne es zu sein: jene Graffitiwände etwa oder aktuell in der Forschung auch so genannte ,Wissensmilieus', die durch die alleinige technisch-planerische Ansiedlung in neuen Industrieclustern oder neuen Stadtteilen entstehen sollen. Im logischen Fehlschluss „kreativer Räume“ wird dieses Dilemma am offensichtlichsten: Ein Raum, zudem physikalisch gedacht als Ausdehnungs- und Containerraum, kann niemals kreativ sein, da Räume keine Eigenschaften besitzen, denn sie sind kein Subjekt, sondern „Subjekt-Objekt“ oder „Produkt“ im obigen Sinne.

Dergleichen gilt für den städtischen Raum. An ihn sind unsere räumlichen (europäischen) Phantasmagorien geknüpft, dass man sich dort frei und ohne (Verwertungs)Zweck muss aufhalten können, soll er lebenswerte Züge haben. Man kann dies wenig geschickt als eurozentrische, altmodische Vorstellung abtun, aber man würde den universellen Zug verkennen, der sich darin verbirgt: ,Europäisch mag der Wunsch sein, Spontaneität in der Öffentlichkeit einer Stadt auszuleben, weswegen hier der Protest gegen anti-urbane Vorhaben besonders groß ist; ,europäisch 'ist aber sicher nicht die Tatsache, dass (städtische) Subjektivitäten die Fiktion von (urbaner) Spontaneität benötigen, wie Beispiele weltweit zeigen - sie sind konstitutiv für jedwede Subjektivität und werden unter Problematisierungszwang offensichtlich (soziale Bewegungen etc.). Diese Fiktion wiederum ist nicht mit einer ,Konstruktion` zu verwechseln, sondern sie ist das Reale unserer Subjektivität im Lacanschen Sinne: die notwendige Stützung unseres Weltzugangs durch etwas Uneinlösbares und Nicht-Verfügbares, das nur durch den Charakter des Unverfügbaren (und ,Unbelegbaren' im positivistischen Sinne) wirkmächtig wird und subjektivische Phantasmagorien stützt. ${ }^{21}$ Dies ist die fundamentale Lagerung von Subjektivität, sie muss alle unsere (insbesondere unbewussten) Vorstellungen begleiten können, sonst werden wir psychotisch in dem Sinne, dass die Vorstellung einer reinen Fremdbestimmung durch Diskurse, Medien, Geld, das Patriarchat oder sonstigen Mächte zum Zusammenbruch führen würde. Das Subjekt wird, in der Regel leiblich, Widerstand dagegen leisten, um „zu überleben“, denn im Imaginären wäre es sonst „tot“ (Lacan 1978: 280; Lacan 1991: 171), weil entsubjektiviert. Auch dies ein Grund, warum bei genau solchen Theorien, die den totalen Gesellschaftszusammenhang annehmen, unvermeidbar „das Andere“ erscheint, ein utopischer Möglichkeitsraum, der diese Vorstellung überhaupt erträglich werden lässt.

Will man also eine auf nicht-instrumenteller Raumerfahrung basierende Utopie wie „Urbanität“ in unseren Bei-

\footnotetext{
${ }^{21}$ Wie z. B. die postmoderne Vorstellung, ,die Welt ${ }^{`}$ sei durch Diskurse ,konstruiert`
} 
spielen rein planerisch-funktional, umsetzen“ (allein dieser Begriff lässt die Unmöglichkeit aufscheinen), dann stürzt die Phantasmagorie des Spontanen, Lebenswerten zusammen, weswegen der Widerstand gegen „kommerzielle Glaspaläste" (oder wie auch immer der Protest konnotiert sein mag) so sicher wie regelmäßig einsetzt. Umgekehrt muss deshalb jede noch so ,instrumentelle' Umsetzung neuer Urbanität genau versuchen, diesen ,spontanen ' Anteil in den Projekten herauszustreichen, gewissermaßen permanent instrumentell auf das Nicht-Instrumentelle hinweisen, weil sonst das Problem zu offenkundig werden würde: Ein Verfahren, das lediglich blanke Verwertungsinteressen erkennen ließe, hätte weder in Politik und Medien noch bei der Bevölkerung eine Legitimation. An Prestigeobjekten lässt sich dies gut verdeutlichen: Ohne die Images, ohne die in den Diskursen und Rationalitäten der Akteure kolportierten Machtbeziehungen, wie diese Orte funktionieren und welches Bild sie abgeben sollen, lassen sich die materialistischen Bedingungen dieser Vorhaben nicht erklären. Man kann insoweit verallgemeinern, dass quasi alle Projekte zur „,neuen Urbanität“ in Deutschland, von Heidelberg über Duisburg, Bremen bis nach Leipzig, eine eigene und vor allem aufwendige Selbstthematisierung im obigen Sinne etablieren. Sie sind ein Indiz der genannten legitimatorischen Engpässe, die sich alleine aus der jeweiligen praktischen Notwendigkeit, Mehrwert durch die Transformation von ökonomischem in symbolisches Kapital zu produzieren, ergeben: denn sie sind als Widerspruch in die Schaffung nicht-instrumenteller Räume praktisch eingelagert.

Ein anschauliches Beispiel des aktiven Raumerschaffens durch solche begleitenden Diskurse (in denen die Räume nach Lefebvre aber nicht aufgehen, die HafenCity ist natürlich kein „diskursiver Raum“ allein) bietet die Geschichte des Projekts selbst. Auch hier setzen mit Anbeginn jene Semantiken ein, die diese Raumproduktion begleiten müssen. Die in der Retrospektive thematisierte Gründung wird ex post einer veränderten geopolitischen Lage zugeschrieben, die einen neuen Raum kreiert habe: ein Europa ohne Grenzen, aber mit neuen Mitten: „Durch den Fall von Berliner Mauer und Eisernem Vorhang hatte sich auch die Rolle Hamburgs in Europa grundsätzlich verbessert: Aus einer Stadt am Ostrand der westlichen Welt war eine Metropole im Herzen eines wieder zusammenwachsenden Kontinents und damit eines beeindruckenden Potenzialraumes [sic] geworden." ${ }^{\text {"22 }}$ Durch die phantasmagorische Semantik des Räumlichen, beeindruckend formuliert im Neologismus „Potenzialraum“, wird hier eine bestimmte Vorstellung wirkmächtig, die Teil jener erwähnten symbolischen Überhöhung ist, die Raumproduktionen pflegen müssen. In diesem Raum wird der kulturelle (und utopische) Mehrwert

\footnotetext{
${ }^{22} \mathrm{http}: / / \mathrm{www} . h a f e n c i t y . c o m / d e /$ ueberblick/hafencity-die-genese-
} einer-idee.html (letzter Zugriff am 10.05.2010). ersichtlich, der hier mit einem bestimmten Sinn eingeführt wird. Er schafft Okkasionalitäten, neue Möglichkeitsräume, die ,nur' erkannt und benannt werden müssen, um die veränderte Lage zu realisieren: als ein neu und aktiv zu kreierendes Territorium, von dem Hamburg und speziell die HafenCity ein Teil geworden sei. Hier zeigt sich mehr als exemplarisch, wie semantisch Raum geschaffen werden kann, wenn es der Begriff nur vermag, kognitiv Raumbilder und damit potenzielle Handlungsspielräume zu imaginieren und Praxis werden zu lassen.

\section{Die Urbanität der HafenCity}

Auch das Urbane rückte von Anbeginn an ins Zentrum des Projekts, als das Herzustellende dieses neuen „Potenzialraums“. Wie bereits erwähnt, entwickelte Henri Lefebvre eine Kritik an instrumentellen Urbanitätsphantasien, die Urbanität eher verdrängen als herstellen. Der Grund liegt in der Antinomie dieser Praxis: die Rahmenbedingungen, unter denen ,es ${ }^{6}{ }^{23}$ das Urbane, eingerichtet werden soll, widersprechen den strukturellen Voraussetzungen für Urbanität im genannten, spontanen' Sinne. Er nennt deshalb solche Bestrebungen „die Illusionen des Urbanismus“, weil die „Urbaniker“ instrumentellen Vorstellungen des Raumes aufsitzen: Der „Urbanismus [will] ein System sein. Er glaubt, eine neue Totalität umfassen, in sich einbeziehen, besitzen zu können. Er nennt sich die moderne Philosophie des Stadtstaates, er glaubt sich durch einen (liberalen) Humanismus gerechtfertigt, weil er eine (technokratische) Utopie rechtfertigt“" (Lefebvre 1972a: 163). Das Problem des Urbanismus (nicht der Urbanität) lässt sich also sehr kurz zusammenfassen: „er behauptet, er könne die urbane Praxis ersetzen“ (Lefebvre 1972a: 163, Hervorhebung im Original).

Die städtebaulichen Modernisierungen sind zwar nicht denkbar ohne bestimmte Images und Leitbilder, die diese anziehend und interessant machen, nicht nur für die Vermarktung restaurierten Wohnraums. Die Art und Weise, wie und welcher Wohnraum, welche Nachbarschaft und welchen Stil eine Wohnung z. B. in der HafenCity besitzen muss, um für eine anvisierte Klientel interessant zu sein, das sind solche „imaginären“ Elemente, die bei Lefebvre mit dem espace vécu angesprochen sind - und seien sie instrumentell und fänden sich in nahezu jedem Zeitungsinserat. Das Hauptinteresse der Analyse muss demnach den urbanen Phantasien gelten, die sich in den Entwürfen und ihren Semantiken lokalisieren lassen. Die Projektgestalter beschreiben dies so: „Urbanität $=$ Die (groß-)städtische Anmutung eines Stadtraums; Urbanität ergibt sich grund-

\footnotetext{
${ }^{23}$ Das Neutrum zeigt genau jenes Nicht-Instrumentelle an: es wird und wird nicht gemacht.
} 
sätzlich aus einer geeigneten städtebaulichen Struktur, aus unterschiedlichen Nutzungen in und außerhalb von Gebäuden sowie aus der Präsenz einer großen Zahl von unterschiedlichen Menschen." ${ }^{\text {24 }}$

Jenseits des anzuerkennenden Bemühens von Seiten der Verantwortlichen, das Großprojekt HafenCity mit solchen Texten inhaltlich aufgeklärt und nachvollziehbar für die Öffentlichkeit zu gestalten, sind es dennoch zwei Aspekte, die in diesem Beispiel ins Auge fallen: die „Anmutung“ des Raumes und die ,große Zahl unterschiedlicher Menschen", die sich dort versammeln sollen. Selbst wenn man davon absieht, dass „Anmutung“ kaum eine Semantik von Unterschichten ist und man sich bereits durch diese Wortwahl elitär abgrenzt (Ghettos, obschon urban, dürften aus dieser Perspektive kaum eine solche Anmutung besitzen), wird daran deutlich, wer sich von solch einem , anmutigen' Stadtraum kulturellen (symbolischen) Mehrwert verspricht: diejenigen Entscheider, die die Definitionsmacht über den urbanen Diskurs in Hamburg innehaben, indem sie etwa durch solche Aussagen präsent sind und die Semantik prägen.

Weiterhin ist der Definitionscharakter aufschlussreich, der mit einem bestimmenden Istgleichzeichen das Thema ,grundsätzlich“ klären möchte, als gelte es, eine allgemeingültige Formel für Urbanität zu finden. Dieser Gestus des Definitorischen, der ein fast funktionales Verständnis des Gesellschaftlichen offenbart, ist ein Indiz für den hier kritisierten instrumentellen Zugriff. Urbanität gibt es weder als Definition noch als planerisches Vorhaben, denn sie lebt vom Gegenteil, dem Nichteingriff und dem ,kreativen Austausch einander fremder Menschen, die eine bestimmte ,unberechenbare' Situation etablieren. ${ }^{25}$ Den Verantwortlichen der HafenCity ist dieser Umstand natürlich bewusst. Aus diesem Grunde zielen viele ihrer Informationen für die interessierte Öffentlichkeit auf dieses ,Unberechenbare` ab, das sich auch und gerade in der HafenCity zeigen soll, um zu bestätigen, dass sie auch urban sei. In gewissem Sinne sind sie geradezu erpicht darauf, Indizien zu sammeln und in ihren Materialien darzustellen, die das Spontane in den Mittelpunkt rücken - wohlwissend, dass Monotonie und Geplantheit dem Grundcharakter und den Intentionen dieses Areals widersprechen würden. Sie legen deshalb großen Wert darauf, dass auch etwas geschehen kann, das nicht geplant war - mit dem ironischen Nebeneffekt, dass selbst abweichendes Verhalten legitimiert und als Beitrag zur Urbanität verstanden wird: „Darüber hinaus zeigen zahlreiche Fotos auch, wie Stadträume durch zusätzliche, zumin-

\footnotetext{
${ }^{24} \mathrm{http} / / / \mathrm{www} \cdot h a f e n c i t y . c o m / d e / q u a r t i e r e . h t m l$ (letzter Zugriff am 12.05.2010)

${ }^{25}$ Und die deshalb auch eine spezifische Gemütshaltung („Blasiertheit") produziert als sozialintegrative Kraft des „Großstädtischen“, worauf Simmel (1995: 121) früh hingewiesen hat.
}

dest nicht ausdrücklich vorgesehene Nutzungen erschlossen werden: Zum Beispiel erklettern Kinder und Jugendliche die verklinkerten Warftwände. Griffmöglichkeiten bieten dabei einzelne vorspringende Backsteine, die in erster Linie zur Gestaltung weithin sichtbarer Muster mit maritimen Motiven dienen." ${ }^{\text {"26 }}$

Die Verantwortlichen goutieren, dass hier jemand, die Grenzen übertritt', also vorgesehene Pfade verlässt und etwas Neues, etwas ,Kreatives' macht. Die Logik des Urbanismus hat sich hier bereits so stark in die Darstellung des Projekts eingeschrieben, dass man von einem Paradebeispiel der ,Selbstkulturalisierung des Städtischen' sprechen kann, und zwar durch den strategischen Verweis auf den urbanen Mehrwert der HafenCity, die Freiräume zulasse und Unvorhergesehenes möglich mache - genau wie es die kultursoziologischen Texte zur Urbanität suggerieren; aber im Subtext dieser Passage wird deutlich, dass dies hier ebenso instrumentell benutzt wird, wodurch es wiederum antinomisch wird.

Im vorherigen Beispiel hingegen bleiben die „unterschiedlichen Menschen“, die von der HafenCity angezogen werden sollen, seltsam unbestimmt, und es ist im Weiteren offenzulegen, wer bzw. welche Milieus hier gemeint sie könnten. Zuerst ist zu vermerken, dass dieser Text eine für die interessierte Öffentlichkeit konzipierte Information und Selbstauskunft darstellt, die sich - als eine der städtischen Gesellschaft verpflichtete Institution - keine selektierende oder gar exkludierende Semantik leisten kann. ${ }^{27}$ Dennoch deutet sich hier bereits eine Einschränkung an, die eine spezifische soziale Exklusion vorwegnimmt: Es heißt nicht „verschiedenster“, sondern „verschiedener" Menschen, was bedeutet, das der Maximalkontrast - Menschen, mit denen man nichts zu tun hat bzw. haben will - hier nicht erscheinen sollen. „Verschiedener“ zielt auf homogenisierte Heterogenität, auf einen Minimalkonsens von Leuten ähnlicher sozialer Lage, die aber individuell genug sind, um sich alter ego gegenüber als different zu fühlen. Die radikale Andersheit oder Fremdheit, in entsprechenden Theorien Grundbedingung für Urbanität, scheint hier entgegen den Aspirationen ungewollt (vgl. Simmel 1995; vor allem Lindner 2008).

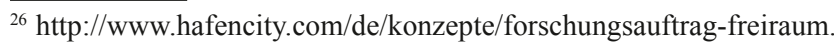
html (letzter Zugriff am 17.05.2010).

${ }^{27}$ In anderen, älteren Projekten, wo der kommerzielle Mehrwert und weniger der urbane im Mittelpunkt stand, wären folgende Einschränkungen bzw. Anschlüsse im Sinne Oevermanns Strukturaler Hermeneutik durchaus denkbar gewesen: „Wir bieten Urbanität ohne störende Elemente“ oder „für die, die sich zu dieser Stadtgesellschaft bekennen", wie es etwa bei der Werbung für gated communities geschieht. Vgl. zur Methodologie Oevermanns allgemein und einführend Oevermann/Allert/Konau et al. (1979) und Oevermann (2002).
} 


\subsection{Die Rolle der Leitbilder}

Das Potenzial des neuen Leitbildes (mittlerweile sind es mehrere „Leitprojekte“ ${ }^{\text {(28) }}$ ) hat sich die Stadt bereits in einem Gutachten bestätigen lassen. Das „Studio UC“ kommt als Beauftragter einer solchen Studie im Januar $2010 \mathrm{zu}$ folgender Einschätzung: ,In der Frage, wie Hamburg sich als zukunftsfähige und wettbewerbsfähige Stadt ausrichten kann, haben sich die Politikfelder bisher auf den Begriff ,Talent' bezogen,der maßgeblich Bestandteil des durch den US-amerikanischen Regionalökonomen Richard Florida entwickelten Konzeptes einer ,Kreativen Klasse“ ist. Die Studie erweitert den Talentbegriff um den des ,Kreativen Milieus‘. Über die mittlerweile übliche Gliederung der Kultur- und Kreativwirtschaft in Teilbranchen hinaus bezeichnen kreative Milieus in dem hier vorliegenden Verständnis Systeme, die jenseits von gesellschaftlichem Stand und sozialer Klasse durch Gruppierungen, Szenen und Atmosphären hervorgebracht werden. Damit werden weiche Standortfaktoren wie die Dynamik von Öffentlichkeiten, Netzwerkbildungen oder Prozesse der Raumaneignung berücksichtigt.“29

Hier fällt sofort die soziale Phantasie der viertletzten Zeile ins Auge: Kreative Milieus, die sich in der HafenCity und andernorts in Hamburg einrichten sollen, würden ,jenseits von gesellschaftlichem Stand und sozialer Klasse“ einen neuen Raum etablieren. Wenn wir den Aspekt beiseite lassen, dass dies eine Auftragsstudie ist und hier kaum sozialwissenschaftlich korrektes Wissen oder Begriffe erwartet werden sollten, so kann man es dennoch als das nehmen, was es ist: eine Studie, die einen bestimmten Jargon pflegt, der anderen (Politik, Planung, Medien) als Legitimationsdiskurs für ihr Vorhaben dient, denn genau dies ist der Zweck solcher Gutachten. Hier manifestiert sich also, als ein weiterer Baustein der Semantik des Posturbanen, der ideologische Anspruch des Projekts, Hamburg zur „Creative City“ zu machen - und vieler weiterer Projekte im Umfeld der „Kreativwirtschaft“: Man kann sagen, dass die Publikationen zum Thema gespickt sind von solchen vormodernen Bildern einer Lebensweise ohne soziale Antagonismen. Dies ist, wie man aus soziologischen Einführungslehrbüchern ersehen kann, aber kein Gesellschafts-, sondern ein Gemeinschaftsbild, also eine vormoderne bis reaktionäre Vorstellung homogener Lebensweisen. Man möchte das Entfremdende, den soziale Schieflagen produzierenden Kapitalismus ,draußen“ lassen und einen Raum schaffen, der naiv gedacht jenseits unserer gegenwärtigen Gesellschaftsordnung steht. Mit anderen Worten: das soziale Elend, die

\footnotetext{
${ }^{28} \mathrm{Vgl}$. abermals http://www.hamburg.de/leitbild-leitprojekte (letzter Zugriff am 20.12.2010).

${ }^{29}$ Einzusehen unter http://www.hamburg.de/contentblob/2052460/ data/gutachten-kreative-milieus.pdf (letzter Zugriff am 21.05.2010).
}

depravierten Klassen, die ,unkreativen“ Migranten mit ihren altmodischen Ökonomien, überhaupt nicht-unternehmerische Selbste (in Anlehnung an Bröckling 2007) haben in diesen Umwelten keine Repräsentation, denn sie würden diese imaginierte soziale Harmonie ,stören'. Stimmt dieser Befund?

\subsection{Materialitäten}

Analysieren wir ergänzend einige populäre bildliche Repräsentationen dazu. ${ }^{30}$

In Abb. 2, verstanden als espace conçu im Lefebvreschen Sinne, der auf einem bestimmten espace vécu und perçu aufbaut und sie dialektisch mitproduziert, finden sich diese Harmonievorstellungen im vorderen Teil der Simulation wieder: Der Innenraum des Zimmers - die milieuspezifischen Sinngebungen der Designer in Form des Magazins „Geo-Special“ im Bücherständer bleiben einmal unthematisiert - weist eine sehr ausgewogene Stimmung und ein ebensolches räumliches Arrangement auf. Hier soll sich der Bewohner der HafenCity nach getaner Arbeit in der Wissensökonomie ausruhen oder geistig mit entsprechender Lektüre erholen können. Solche Interieurs weisen gemäß den Studien der Wohnsoziologie ${ }^{31}$ auf gehobenere, akademisch ausgebildete Milieus hin, da es dort zum Beispiel keine ,kleinbürgerlichen“ Familienfotos oder „Gelsenkirchener Barock“ gibt, sondern eher Abstrakt-Kulturelles wie die Vasen und Plastiken im Regal und auf dem Boden. Der „Loft Chair“" ist ebenso wenig ein Sitzmöbel eines Arbeiters, sondern in gewissem Sinne die verdinglichte habituelle Antithese. Interessanterweise sieht man aber auch keine bildungsbürgerliche Bücherwand mit ,Klassikern“ oder ähnlichem, weswegen sich die Hinweise auf das ,Kreative Milieu' verdichten, das hiermit angesprochen werden soll: Dieses versteht Wissen in Form unhandlicher Materialitäten wie Bücher als Ballast und favorisiert digitales Know-how. Wenn überhaupt, so arbeitet man mit Projektskizzen, um die es sich symbolisch beim DIN-A4-Hefter auf der Fußablage in der Mitte des Zimmers handeln könnte.

Der Verdacht, dass dies eine räumliche Repräsentation für die „kreative Klasse“ sein könnte, lässt sich erhärten, indem zusätzlich auf den Bildhintergrund geachtet wird: Hier erscheint die alte, harte Arbeitswelt des 19. und 20. Jahrhunderts als anregende Kulisse im Sonnenuntergang, wodurch sie zum einen so weit wie möglich vom Bewohner entfernt ist (das Wasser trennt beide Welten nahezu ultimativ), zum anderen aber noch eine Reminiszenz, ein postmodernes Zitat

\footnotetext{
${ }^{30}$ Die methodologischen Ausführungen zu einer Bild- und Videoanalyse würden den Rahmen dieser Arbeit sprengen, sie muss an anderer Stelle erfolgen. Vgl. einführend dazu Bohnsack (2007: 73 ff.) und Bohnsack (2009: 60 ff.).

${ }^{31} \mathrm{Vgl}$. zu einer Bourdieuschen Herangehensweise wie hier angestrebt z. B. Katschnig-Fasch (1998).
} 


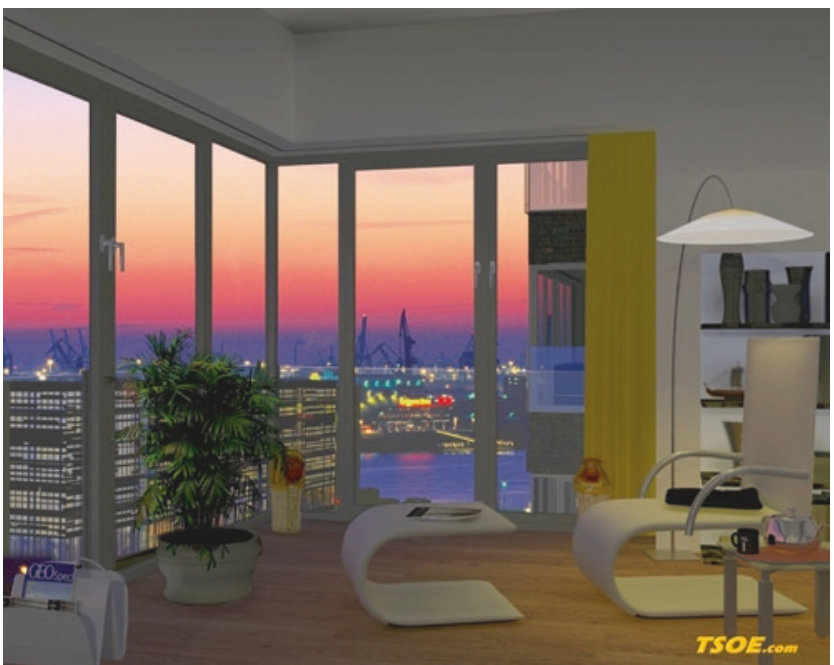

Abb. 2 Computersimulation eines Lofts für die HafenCity. (Quelle: http://www.tsoe.com/neu/hafencity.htm (letzter Zugriff am 15.05.2010))

der Arbeitswelt darstellt, und damit durchaus den Selbstthematisierungen der „Kreativen“ entspricht. Diese „nennen“ es nur noch Arbeit, was sie in Projekten und durch Netzwerke schaffen (Friebe/Lobo 2006). Sie wollen sich nicht mehr als fordistisch angestellte Bevölkerung sehen, sondern begreifen ihre (durchaus selbstausbeuterischen) Arbeitsverhältnisse als Freiheit von alten Zwängen (gegen nine-to-fiveliving, konservative dresscodes im Büro etc.). Sie pflegen die alte Industriekulisse aber als entwordenes Schauspiel, das ihnen wiederum zur Produktion kulturellen Mehrwerts dient: nicht nur, dass sich die Kreativökonomie vornehmlich in alten Industriearealen niederlässt und deswegen gerade die innerstädtischen Brachen aufsucht (die technisch selbstverständlich auf den neuesten Stand gebracht wurden), welche durch ihre nun in "Premiumlage“ liegenden Areale die perfekten Flächen dafür darstellen. Zudem liefern diese Kulissen Möglichkeiten, Altes mit Neuem ,kreativ' zu verbinden, ein kultureller habit der Kreativwirtschaft: Deswegen gehen auch die Eigennamen der Start-ups wie ganzer Ensembles gerne eine solche semantische Verbindung zwischen alt und neu ein, ein postmodernes Pastiche im Sinne Jamesons (1996: 17): „Pastiche is, like parody, the imitation of a peculiar or unique, idiosyncratic style, the wearing of a linguistic mask, speech in a dead language. [...] Modernist styles thereby become postmodernist codes [...] a field of stylistic and discursive heterogeneity without a norm" - „Kulturfabriken“ oder „Planwerkstätten“ geben darüber Auskunft.

Dieses Muster kultureller Produktion (und letztlich der Selbstthematisierung von ,Kreativen' über ,Kreative') zieht sich durch nahezu alle Schilderungen, die den Prozess begleiten. Sie sind dadurch eine interessante Auskunftsquelle. Besonders in Computersimulationen zum Zukünf-

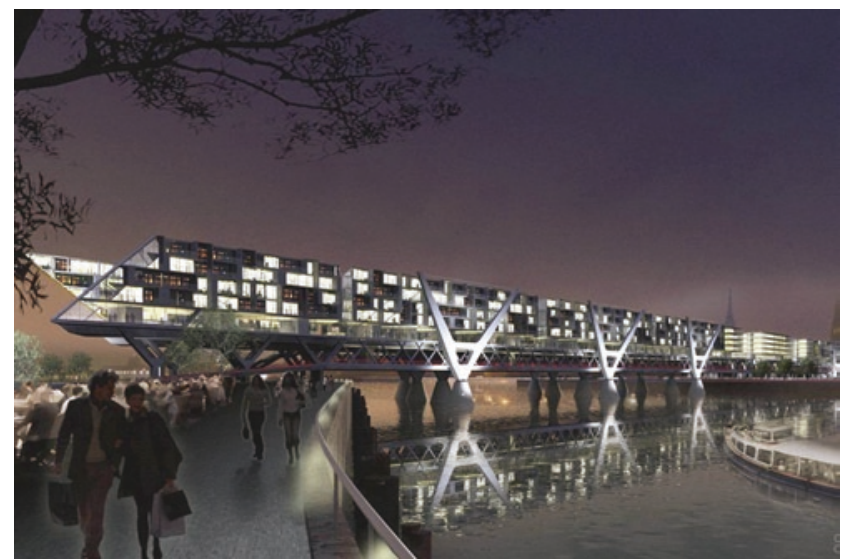

Abb. 3 Die sogenannte Living Bridge. (Quelle: dpa; BRT Architekten dpa/lno, http://www.livingbridge-hamburg.de/nacht.html (letzter Zugriff am 23.02.2011))

tigen dieser Lebens- und Arbeitswelten wird dies deutlich, da sie eine perfekte Manifestation der Gesellschaftsbilder ihrer Produzenten liefern, denn das ist der Zweck solcher Produktionen: Sie sollen ein (noch) utopisches Szenario anzeigen, das dennoch soweit realistisch sein muss, dass es mit den gegenwärtigen Mitteln umsetzbar erscheint. In dieser Dialektik befinden sich natürlich auch solche Simulationen zu urbanen Zukünften, da sie einerseits auf Gegebenes und Machbares abzielen (der konkrete Anlass für solche Entwürfe ist ja gerade dieser Imperativ), andererseits aber auch gesellschaftliche Wünsche bedienen müssen. Es stellt sich an dieser Stelle aber vor allem die Frage: wessen Wünsche?

Die vorgesehene, spektakuläre Brücke (vgl. Abb. 3), die die HafenCity mit den südlichen Stadtteilen Wilhelmsburg und Veddel verbinden soll, ist die Living Bridge. Ihre raumprägenden Funktionen (Wohnen, Arbeiten, Flanieren, Konsumieren innerhalb der Brücke) als Verbindung schaffender espace conçu kann als Paradebeispiel für eine Raumproduktion im Lefebvreschen Sinne verstanden werden. Die Diskussion um diese Brücke selbst ist ein Zeugnis umkämpfter Urbanität, wie sie auf deren Internetseite nachverfolgt werden kann. ${ }^{32}$ Zwar ist der epace perçu, der damit geschaffen werden soll, durchaus subtiler als auf den ersten Blick ersichtlich (die Etablierung einer urbanen Lebendigkeit durch Aufenthalts-, Arbeits-, ästhetische und Vergnügungsfunktionen im Sinne einer speziellen urbanen Praxis, die dort stattfinden soll), sein espace veçu hingegen kann kaum übersehen werden und ist hier besonders aufschlussreich: Vorbild für all dies sind bewohnte Brücken wie der Ponte Vecchio in Florenz, jene Bauwerke aus der Zeit der

\footnotetext{
${ }^{32}$ Leider wurde im Zuge der Abfassung des Artikels die Website http://www.belebte-bruecke.de abgeschaltet und auf http://www. livingbridge-hamburg.de umgeleitet. Hier wurden - als würde das Geschriebene in actu bestätigt - alle kritischen Stimmen dazu entfernt und stattdessen eine Werbeseite erstellt.
} 


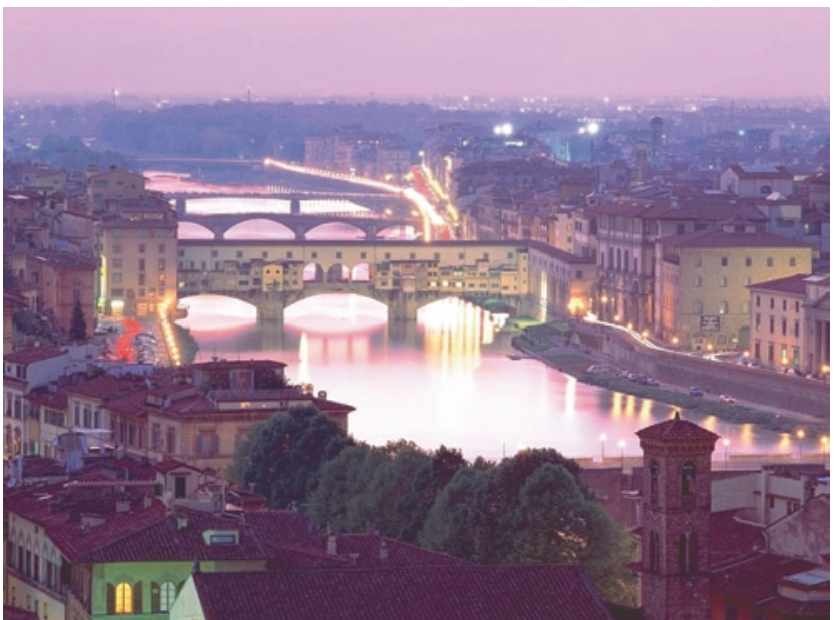

Abb. 4 Der Ponte Vecchio in Florenz. (Quelle: http://italywallpaper. info/bulkupload/ItalyWallpapers/Ponte\%20Veccio.jpg (letzter Zugriff am 23.02.2011))

Frührenaissance, die dort für ein ,typisch südländisches Flair" sorgten und dafür immer noch Sinnbild sind - wenn auch im inhaltlich anderen Sinne als es die Projektion zur HafenCity hier anvisiert: Der „Traum eines hanseatischen Ponte Vecchio“33 orientiert sich paradoxerweise also an Aufenthaltsqualitäten von Bauwerken im , alteuropäischen “ Sinne (buntes unkontrollierbares Treiben), deren Arbeitswelten eindeutig vormodern waren (wildes Durcheinander von Ständen, Läden etc., Gestank und ,dreckige‘ Ökonomien wie Gerber; vgl. Abb. 4).

Es ist eine gewisse Ironie, wenn man als zentrales Sinnbild für ein hypermodernes, vielleicht posturbanes Projekt auf einen geradezu klassischen Bau der mittelalterlichen europäischen Stadt zurückgreift. Wenn man aber den kulturellen Mehrwert, den man im espace vécu durch die semantische Verknüpfung von europäischer Stadt und anvisiertem Projekt gewinnt, als notwendige Strategie im hier beschriebenen Sinne begreift, dann bekommt der Verweis einen durchaus realen Hintergrund und es wird verständlich, warum solche Verknüpfungen überhaupt notwendig sind: einzig wegen des Fehlens jener Form europäischer Urbanität in der HafenCity, die aber dennoch - anders ist das Beispiel kaum zu verstehen - die Phantasmagorien der Verantwortlichen antreibt.

Hier ist nichts gegen den anregenden Entwurf und dessen Ansinnen an sich gesagt, aber was diese Versinnbildlichung für die urbane Praxis der HafenCity zeigt, ist jenes Problem, das hier als „Antinomie des Urbanismus“ geführt wird: Die Unmöglichkeit, etwas Spontanes und Ungeplantes mittels Planung und Rationalität herzustellen. Es mag Urbanität anvisiert sein, das Ergebnis wird Posturbanität sein. Dies

\footnotetext{
${ }^{33}$ Vgl. Imagetrailerunterhttp://www.youtube.com/watch?v=HdS019ORmZg (letzter Zugriff am 21.05.2010).
}

kann als Grunddilemma moderner Stadtplanung verstanden werden, sofern sie sich dem Prinzip der Schaffung von Urbanität als rationalistischem Dispositiv verschrieben hat. Diese Praxis nenne ich posturban, weil sie im Rekurs auf das europäische Paradigma ihre eigene Antithese hervorbringt, deren symbolischer Gehalt nur noch als Mythos die technischen Projekte beseelt, aber vermutlich nie als authentischer Erfahrungsraum tatsächliche urbane Praxis mit allen ihren (auch missleidigen) Konsequenzen möglich macht. An einem letzten exemplarischen Dokument soll deshalb diese Antinomie nochmals verdeutlicht werden.

Es wird aus einem Protokoll eines Treffens mit Verantwortlichen der Hamburger Planungsbehörden zitiert, das unsere Forschergruppe angefertigt hat. „Frau X. beschränkt sich auf die ,landläufige Vorstellung', dass alles, was an städtischem Leben sichtbar ist, Urbanität ausmacht. Voraussetzung dieses Aspektes von Urbanität ist Angebot und Nachfrage. So kann, laut X., die Entwicklung von Urbanität punktuell oder strukturell ermöglicht werden. Einrichtungen oder auch Anziehungspunkte schaffen punktuelle Voraussetzung für Urbanität, wohingegen sich Anziehungsgebiete durch eine ,Szene', die Vermittlung eines Lebensgefühls und/oder besondere soziale Vernetzung auszeichnen. Zur Installation eines solchen Gebietes ist auch ein kulturelles Angebot notwendig, das sich zum Teil bereits in organisierten Lesungen oder Freiluftkonzerten niederschlägt" (Interview mit X., Büros der Stadt Hamburg, am 28.03.2010).

Es sei ausdrücklich darauf verwiesen, dass es sich hier nicht um Mitarbeiter der HafenCity AG handelt, die durchaus differenziertere Argumentationen aufbieten. Aber an dieser Sentenz zeigt sich das Dilemma in seiner ganzen Breite: Dem technizistischen Jargon der Planungsbehörden verpflichtet, wird hier versucht, über Urbanität, den flüchtigen Signifikanten, im Modus des Urbanikers (Lefebvre) Auskunft zu geben. Dieses Beispiel ist nur eines von vielen, dennoch oder gerade deswegen kann es als paradigmatisch angesehen werden: Beim Versuch, den Diskurs über das Urbane, den die Person als auskunftsgebendes Subjekt auf Nachfrage hin führen muss, in den ihr möglichen Semantiken auszudrücken, gerät das Unterfangen bereits im ersten Satz unter Beschuss. Die „landläufige Vorstellung“ soll als imaginärer Herrensignifikant Autorität sichern, wo noch keine waltet: Man sagt, was ,man“ sagt. Dieses erste Unvermögen, von selbst Auskunft über das Urbane geben zu können und statt dessen auf eine imaginierte und gebilligte Autorität verweisen zu müssen, wird weiters gesteigert, wenn es heißt, das „Sichtbare“ städtischen Lebens bilde Urbanität ab. Der Versuch also, dem bereits anfänglich entglittenen Begriff ein wenig Substanz beizusteuern, etwas, das man messen oder wenigstens sehen könne, um dem positivistischen Weltbild der Planung das Manna zu liefern, scheitert abermals, wenn die hier eingeführten Kriterien für Urbanität zu Grunde gelegt werden: Das Flüchtige, Nicht-Gesehene 
des ,Zwischen“ urbaner Interaktion ist ja gerade die urbane Erfahrung.

Die Insuffizienz auch in raumsoziologischer Hinsicht wird mit dem nächsten Satz gewahr. Urbanität wird hier als punktueller initial spark gedacht, als etwas, das man , setzen' müsse wie ein Feuer oder eine Innovation, die sich ausbreite, hat sie einmal Fuß gefasst. Man kann es der Person nicht vorwerfen, aber hier spricht die Raumsemantik des Planungsdiskurses der 1970er Jahre: Raum, das sei der Container oder die „Registrierplatte“ im Sinne von Hartke (1962: 116), auf welcher sich, einem Schaltkreis nicht unähnlich, urbanes Leben durch „Anziehungsgebiete“ einstellen soll. Man kann diese Interviewpassage noch wesentlich intensiver interpretieren, für unsere Zwecke soll ein letzter Hinweis reichen: Die „Installation“ (letzter Satz) solcher Gebiete komprimiert das erwähnte Dilemma auf genau jenen einen Begriff, der die Unmöglichkeit, Urbanität „herzustellen“, deutlich macht, indem er seine Antithese modelliert: Sie, die Urbanität, ist als authentische Erfahrung das genaue Gegenteil von dem, was aus dieser Phantasmagorie spricht, nämlich das Nicht-Installierte bzw. Nicht-Instrumentelle.

\section{Resumée}

Alle angeführten Beispiele sollten Aspekte einer „Raumproduktion" im Sinne Lefebvres analysieren, die das Urbane (oder Phantasien über das Urbane) als konkrete Praxis begleiten müssen. Raumproduktionen nach Lefebvre inkorporieren drei Register: Hier ,spricht' eine gesellschaftliche Praxis, die einen bestimmten wahrgenommenen Raum thematisiert, etwa die Vorstellung aus (die auch in der Praxis ihre Erfahrung haben muss), dass ,Anziehungspunkte durch eine ,Szene““ (letztes Beispiel) für urbane Neubaugebiete wichtig und zentral seien. Wie diese nun konkret werden, auf welcher räumlichen Wissensform als vorgestellte Relationen im Raum sie beruhen, das ist wiederum Teil des conçu, der darin ebenso die kulturellen und historischen Lagerungen dieser Wissensform darstellt. Und, das ist das Entscheidende, will man die Latenzen solcher Bilder und der Semantiken verstehen, beinhalten solche Raumrepräsentationen auch im espace vécu, also im nicht direkt zeichenhaft repräsentierbaren Anteil, die „Raumphantasien“, die diese Images und Begrifflichkeiten notwendig begleiten. Ebenso verhält es sich natürlich mit dem Begriff der Urbanität, der meist in eher technischer Hinsicht definiert wird, aber einen imaginären Mehrwert hat, der dann in solchen Definitionen meist unbeholfen als Verweis auf etwas ,größeres', irrational Gegebenes fungieren muss. Deshalb nannte ich die Konsequenz daraus für die städtische Praxis „Posturbanität“. Dabei sollte deutlich geworden sein, dass Urbanität im Lefebvreschen Sinne exakt das Gegenteil dessen ist, nämlich eine spezifisch städtische Erfahrung der
Offenheit, Abwechslung und Differenz, die sich nur einstellt, wenn man von ihr lässt. Das untersuchte Beispiel HafenCity wird deswegen genau dann zur Ideologie, wenn es ,von den Urbanikern als repräsentativ für die urbane Ordnung akzeptiert wird, obwohl es absondert“" (Lefebvre 1972a: 144).

Open Access Dieser Artikel unterliegt den Bedingungen der Creative Commons Attribution Noncommercial License. Dadurch sind die nichtkommerzielle Nutzung, Verteilung und Reproduktion erlaubt, sofern der/die Originalautor/en und die Quelle angegeben sind.

\section{Literatur}

Belina, B. (2007): From Disciplining To Dislocation. Area Bans in Recent Urban Policing in Germany. In: European Urban and Regional Studies 14, 4, 321-336.

Benjamin, W. (1991 [1935]): Paris, die Hauptstadt des XIX. Jahrhunderts. In: Benjamin, W. (Hrsg.): Das Passagen-Werk. Gesammelte Schriften Band V.1. Frankfurt am Main.

Berking, H.; Löw, M. (2008): Die Eigenlogik der Städte: Neue Wege für die Stadtforschung. Frankfurt am Main.

Bohnsack, R. (2007): Rekonstruktive Sozialforschung. Opladen.

Bohnsack, R. (2009): Qualitative Bild- und Videointerpretation. Opladen.

Bourdieu, P. (1999): Sozialer Sinn. Kritik der theoretischen Vernunft. Frankfurt am Main.

Bröckling, U. (2007): Das unternehmerische Selbst. Soziologie einer Subjektivierungsform. Frankfurt am Main.

Bruns-Berentelg, J. (2008): Interview. In: Standort - Zeitschrift für Angewandte Geographie 32, 2, 40-44.

Florida, R. (2004): The rise of the creative class ... and how it's transforming work, leisure, community, and everday life. New York.

Foucault, M. (1999): In Verteidigung der Gesellschaft. Frankfurt am Main.

Friebe, H.; Lobo, S. (2006): Wir nennen es Arbeit. Die digitale Boheme oder: Intelligentes Leben jenseits der Festanstellung. München.

Hartke, W. (1962): Die Bedeutung der geographischen Wissenschaft in der Gegenwart. In: Tagungsberichte und Abhandlungen des 33. Deutschen Geographentages in Köln 1961. Wiesbaden, 113-131.

Harvey, D. (1989): The Condition of Postmodernity. An Enquiry into the Origins of Cultural Change. Oxford.

Harvey, D. (1991): Geld, Zeit, Raum und die Stadt. In: Wentz, M. (Hrsg.): Stadt-Räume. Raum und Zeit in der metropolitanen Entwicklung. Frankfurt am Main, 149-168.

Harvey, D. (2007): Räume der Neoliberalisierung. Hamburg.

Hörning, K.; Gerhard, A.; Michailow M. (1990): Zeitpioniere. Flexible Arbeitszeiten - neuer Lebensstil. Frankfurt am Main.

ILS - Institut für Landes- und Stadtentwicklungsforschung (2008): Kreative Ökonomie und Kreative Räume: Kultur- und Kreativwirtschaft in der integrierten Stadtentwicklung. Endbericht im Auftrag des Ministeriums für Bauen und Verkehr des Landes Nordrhein-Westfalen. Dortmund. Online unter: http://www.ilsforschung.de/images/stories/publikationen/kreative_raeume.pdf (letzter Zugriff am 15.05.2010).

Jameson, F. (1996): Postmodernism, or, the cultural logic of late Capitalism. London/New York.

Katschnig-Fasch, E. (1998): Möblierter Sinn. Städtische Wohn- und Lebensstile. Wien.

Köppen, B. (2008): Reurbanisierung als Hoffnung der Städte im demographischen Wandel? In: Maretzke, S. (Hrsg.): Städte im demografischen Wandel. Wesentliche Strukturen und Trends des demografischen Wandels in den Städten Deutschlands. Wiesbaden, 31-40. = Materialien zur Bevölkerungswissenschaft, Bd. 125. 
Köppen, B. (2011): Ohne Einwohner keine Stadt: Die Hoffnungsformel „Reurbanisierung“ für die deutschen Städte zwischen Wunsch und Wirklichkeit. In: Gehler, M. (Hrsg.): Die Macht der Städte. Hildesheim, 285-296. = Historische Europa-Studien, Bd. 4.

Lacan, J. (1978): Die symbolische Ordnung. In: Lacan, J. (Hrsg.): Das Seminar. Buch I. Olten/Freiburg, 278-294.

Lacan, J. (1991): Subversion des Subjekts und Dialektik des Begehrens im Freudschen Unbewußten. In: Lacan, J. (Hrsg.): Schriften II. Weinheim, 165-204.

Lefebvre, H. (1972a): Die Revolution der Städte. München.

Lefebvre, H. (1972b): Das Alltagsleben in der modernen Welt. Frankfurt am Main.

Lefebvre, H. (1974): Kritik des Alltagslebens. Band 1. München.

Lefebvre, H. (1975): Kritik des Alltagslebens. Band 2. München.

Lefebvre, H. (1978): Einführung in die Modernität. Zwölf Präludien. Frankfurt am Main.

Lefebvre, H. (2000): The production of space. Oxford.

Lindner, R. (2008): The virtue of diversity. Unveröffentlichtes Typoskript zur Tagung in der HafenCity über „Urban Planning“ im März 2008

Löw, M. (2009): Soziologie der Städte. Frankfurt am Main.

Mai, R.; Schlömer, C. (2007): Erneute Landflucht? Wanderungen aus dem ländlichen Raum in die Agglomerationen. In: Zeitschrift für Bevölkerungswissenschaft 32, 3-4, 713-742.

Mitchell, D. (2000): Cultural Geography. A Critical Introduction. Oxford.

Oevermann, U. (2002): Klinische Soziologie auf der Basis der Methodologie der objektiven Hermeneutik - Manifest der objektiv hermeneutischen Sozialforschung. Online: http://publikationen. ub.uni-frankfurt.de/volltexte/2005/540/pdf/ManifestWord.pdf (letzter Zugriff am 20.12.2010).
Oevermann, U.; Allert, T.; Konau, E.; Krambeck, J. (1979): Die Methodologie einer „objektiven Hermeneutik“ und ihre allgemeine forschungslogische Bedeutung in den Sozialwissenschaften. In: Soeffner, H.-G. (Hrsg.): Interpretative Verfahren in den Sozial- und Textwissenschaften. Stuttgart, 352-434.

Reckwitz, A. (2009): Die Selbstkulturalisierung der Stadt. Zur Transformation moderner Urbanität in der „,creative city“. In: Mittelweg $3618,2,2-34$.

Schmid, C. (2005): Stadt, Raum und Gesellschaft. Henri Lefebvre und die Theorie der Produktion des Raumes. Stuttgart.

Simmel, G. (1995): Die Großstädte und das Geistesleben. In: Simmel, G. (Hrsg.): Gesamtausgabe. Band 7. Frankfurt am Main, 116-131.

Trinczek, R. (1999): „Es gibt sie, es gibt sie nicht, es gibt sie, es ...“ - Die Globalisierung der Wirtschaft im aktuellen sozialwissenschaftlichen Diskurs. In: Schmidt, G.; Trinczeck, R (Hrsg.): Globalisierung. Ökonomische und soziale Herausforderungen am Ende des zwanzigsten Jahrhunderts. Baden-Baden, 55-75. = Soziale Welt, Sonderband 13.

Wacquant, L. (2008): Urban Outcasts. A comparative Sociology of advanced marginality. Cambridge.

Wilson, D. (2009): Racialized poverty in U.S. cities: Towards a refined racial economy perspective. In: The Professional Geographer 61, 2, 139-149.

Žižek, S. (2009): Auf verlorenem Posten. Frankfurt am Main. 